\title{
A PROOF OF TWO FUNDAMENTAL THEOREMS ON LINEAR TRANSFORMATIONS IN HILBERT SPACE, WITHOUT USE OF THE AXIOM OF CHOICE
}

\section{BARSOTTI}

The definitions and notations used in this paper may be found in the volume Linear transformations in Hilbert space by M. H. Stone, ${ }^{1}$ which work I shall quote as $\mathrm{S}$.

Many of the consequences of S, chaps. 4-9, are based on the existence of transformations of a particular kind called projections ( $S$, Definition 2.16), and such existence is a consequence of $\mathrm{S}$, Theorem 1.23, whose proof is based on $\mathrm{S}$, Theorem 1.18. The last one is a particular case of a Hausdorff theorem ${ }^{2}$ on abstract spaces, and its proof depends upon the axiom of choice. I shall show, without use of that axiom, that "every closed linear manifold $\mathfrak{M}$ in the Hilbert space $\mathfrak{S}$ has an orthogonal complement $\mathfrak{S} \ominus \mathfrak{M}$." The consistency of $\mathrm{S}$, Definition 2.16 will thus be ensured.

Another theorem based on the axiom of choice is $\mathrm{S}$, Theorem 2.25. This one I shall prove as well without the aid of the axiom.

It is necessary to make clear the meaning of the word "closed" applied to a subset $\mathfrak{M}$ of the Hilbert space $\mathfrak{W}$.

Let $f$ be a point of $\mathfrak{S} . f$ is said to be a point of accumulation of $\mathfrak{M}$ if for every real number $\epsilon>0$ there exists a point $g \neq f$ of $\mathfrak{M}$ such that $|g-f|<\epsilon . \mathfrak{M}$ is said to be closed when every point of accumulation of $\mathfrak{M}$ is a roint of $\mathfrak{M}$.

An alternative definition is the following: $f$ is a point of accumulation of $\mathfrak{M}$ if there exists an infinite sequence $\left\{g_{i}\right\}$ of points of $\mathfrak{M}$ such that $g_{i} \neq f(i=1,2, \cdots), \lim _{i \rightarrow \infty} g_{i}=f ; \mathfrak{M}$ is said to be closed when every point of accumulation of $\mathfrak{M}$ is a point of $\mathfrak{M}$.

It is well known that, if the axiom of choice is accepted, these two definitions are equivalent; if not, a subset $\mathfrak{M}$ closed according to the first definition is also closed according to the second one, but the converse is not necessarily true. Therefore, we shall assume the first meaning of the word "closed" to be the correct one.

In the logical development of the theory, Theorems 1.3 to 1.14 of $\mathrm{S}$ can be stated and proved: it must be remarked that in the proof of $S$, Theorem 1.13, a well determined orthonormal set $\left\{\phi_{n}\right\}$ is found. Therefore, when a sequence $\left\{f_{n}\right\}$ is given, it is possible to

Received by the editors March 31, 1947.

1 Amer. Math. Soc. Colloquium Publications, vol. 15, 1932.

${ }^{2}$ Hausdorff, Grundzïge der Mengenlehre, Theorem VIII, p. 273. 
choose, among the generally infinitely many orthonormal sets which determine the same linear manifold and the same closed linear manifold as $\left\{f_{n}\right\}$, a particular one, which will be called the orthonormal set attached to $\left\{f_{n}\right\}$. Let $\mathfrak{M}$ now be a closed linear manifold in $\mathfrak{W}, \mathfrak{S}$ a relatively open subset of $\mathfrak{M}$, in brief an $O$-set in $\mathfrak{M}$, that is, such that for every $f$ of $\subseteq$ there exists a real $\epsilon>0$ such that if $g$ is in $\mathfrak{M}$ and $|g-f| \leqq \epsilon$, then $g$ belongs to $\widetilde{S}$. The set consisting of the points of $\subseteq$ and of its points of accumulation is the closure of an $O$-set of $\mathfrak{M}$, in brief a $C$-set in $\mathfrak{M}$, and is still a subset of $\mathfrak{M}$.

The sphere with center $\mathbb{S}$ and radius $r>0$ is the set consisting of the points $g$ of $\mathfrak{S}$ such that $|g-f|<r$ for every $f$ of $\mathfrak{S}$, whenever such set is not empty. The same definition holds when $\subseteq$ is a $C$-set in $\mathfrak{M}$; the set consisting of the points of sphere and of its points of accumulation will be named a closed sphere.

Recalling the definition of diameter of a set in a metric space, ${ }^{3}$ it is easy to prove the following lemma.

(1) Lemma. Let $\subseteq$ be an O-set or a $C$-set in the closed linear manifold $\mathfrak{M}$. Then a necessary and sufficient condition that a closed sphere of center $\subseteq$ and radius $r$ exist is that $r \geqq d$, where $d$ is the diameter of $\mathfrak{S}$; and if $d_{1}$ is the diameter of the sphere, then $d \leqq d_{1} \leqq 2 r$. A sphere (a closed sphere) is an O-set (a C-set) in $\mathfrak{S}$.

(2) Lemma. Let $\left\{\Im_{m}\right\}$ be a sequence of $C$-sets in a closed linear manifold $\mathfrak{M}$, such that $\mathfrak{S}_{m+1} \subseteq \Im_{m}(m=1,2, \cdots)$. If $d_{m}>0$ is the diameter of $\mathfrak{S}_{m}$ and $\lim _{m \rightarrow \infty} d_{m}=0$, then there exists one (and not more than one) point belonging to every $\mathfrak{S}_{m}{ }^{4}$

Proof. Let $\mathfrak{S}_{m}^{(1)}$ be the closed sphere with centre $\mathfrak{S}_{m}$ and radius $d_{m}$ (1), and $\mathfrak{S}_{m}^{(2)}$ the intersection of $\Im_{1}^{(1)}, \Im_{2}^{(1)}, \ldots, \Im_{m}^{(1)}$. Then $\Im_{m}^{(2)}$ is a non-empty $C$-set in $\mathfrak{S}$, since $\mathfrak{S}_{m} \subseteq \Im_{m}^{(2)}$, and the relation $\mathfrak{S}_{m+1}^{(2)} \subseteq \Im_{m}^{(2)}$ is satisfied for $m=1,2, \cdots$.

Let $\left\{f_{m}\right\}$ be a denumerably infinite set of points dense in $\mathfrak{S}$ (whose existence is asserted by $S$, Postulate $D, p .3$ ) fixed once for all. If $\left\{f_{m}^{(1)}\right\}$ is the sequence of the elements of $\left\{f_{m}\right\}$ belonging to $\mathfrak{S}_{1}^{(1)}$, $\left\{f_{m}^{(1)}\right\}$ is surely not empty, and may be easily shown to be dense in $\widetilde{S}_{1}^{(1)}=\Im_{1}^{(2)}$.

The sequence $\left\{f_{m}^{(2)}\right\}$ formed with the elements of $\left\{f_{m}\right\}$ belonging to $\mathfrak{S}_{2}^{(2)}$ is also not empty, is dense in $\Im_{2}^{(2)}$, and is a sub-sequence of $\left\{f_{m}^{(1)}\right\}$;

' Kuratowski, Topologie, vol. 1, Warsaw, 1933, chap. 15, III, p. 85.

${ }_{4}^{4}$ This is a particular case of a theorem stated by Saks (Theory of the integral, 1937, chap. 2, Theorem 9.1, p. 54) for more general spaces, with the aid of the axiom of choice. 
and the same properties are valid for the sequence $\left\{f_{m}^{(n)}\right\}$ chosen with respect to $\mathfrak{S}_{n}^{(2)}(n=1,2, \cdots)$. Let $f_{m(n)}$ be the first element, in the ordering of $\left\{f_{m}\right\}$, which belongs to $\left\{f_{m}^{(n)}\right\}$. If $\epsilon>0$ is given, there exists an integer $M$ such that $d_{M}<\epsilon / 2$, and if $n_{1}, n_{2}>M$, it follows that

$$
\left|f_{m\left(n_{1}\right)}-f_{m\left(n_{2}\right)}\right| \leqq 2 d_{M}<\epsilon .
$$

Therefore, for S, Postulate E, p. 3, there exists an $f$ such that $\lim _{n \rightarrow \infty} f_{m(n)}=f$. Since, for $n>M, f_{m(n)}$ belongs to $\Im_{M}^{(1)}$, and $\Im_{M}^{(1)}$ is closed, $f$ belongs to $\mathfrak{S}_{M}^{(1)}$ as well, and then it belongs to every $\mathfrak{S}_{m}^{(1)}$.

When $g$ is an arbitrarily chosen element of $\Im_{M}$, we have

$$
|g-f| \leqq 2 d_{M}<\epsilon,
$$

and therefore $f$ is a point of accumulation of $\mathfrak{M}$ according to our definition. $\mathfrak{M}$ being closed, we conclude that $f$ belongs to $\mathfrak{M}$, and consequently to every $\mathfrak{S}_{m}$. The uniqueness of $f$ is easily proved, Q.E.D.

(3) Theorem. Let $\mathfrak{M}$ be a closed linear manifold, $f$ a point of $\mathfrak{S}$. Then there exists a (unique) point $g_{0}$ of $\mathfrak{M}$ such that $\left|f-g_{0}\right|<|f-g|$ for every $g \neq g_{0}$ of $\mathfrak{M}$.

Proof. When $f$ belongs to $\mathfrak{M}$, it is $g_{0}=f$. Otherwise, let $d>0$ be the lower bound of the values of $|f-g|$ when $g$ ranges over $\mathfrak{M}$, and let $\mathfrak{S}_{m}^{(0)}$ be the (non-empty) set consisting of the elements $g$ of $\mathfrak{M}$ such that $d \leqq|g-f|<d+1 / m(m=1,2, \cdots)$. When $g_{1}$ belongs to $\Im_{m}^{(0)}$ and $g_{2}$ to $\mathfrak{M}$, it follows from $\left|g_{1}-g_{2}\right|<\epsilon$ that

$$
\left|g_{2}-f\right| \leqq\left|g_{2}-g_{1}\right|+\left|g_{1}-f\right|<\left|g_{1}-f\right|+\epsilon,
$$

so that if we choose $\epsilon<d+1 / m-\left|g_{1}-f\right|$, the relation $\left|g_{2}-f\right|$ $<d+1 / m$ holds, showing that $\mathfrak{S}_{m}^{(0)}$ is a 0 -set in $\mathfrak{M}$. Therefore the set $\widetilde{S}_{m}$ consisting of the points $g$ of $\mathfrak{M}$ for which the relation

$$
d \leqq|g-f| \leqq d+1 / m \quad(m=1,2, \cdots)
$$

holds, is a $C$-set in $\mathfrak{M}$, and we have, of course, $\mathfrak{S}_{m+1} \subseteq \mathfrak{S}_{m}(m=1$, $2, \cdots)$. Let now $g_{1}, g_{2}$ be distinct elements of $\mathfrak{S}_{m}$. We have: $\left|g_{2}-f\right|^{2}$ $=\left|g_{1}-f\right|^{2}+\left|g_{2}-g_{1}\right|^{2}+2 \Re\left[\left(g_{1}-f\right) \times\left(g_{2}-g_{1}\right)\right] \leqq(d+1 / m)^{2}$, where $\Re[x]$ is the real part of $x$, and also $-\left|g_{1}-f\right|^{2} \leqq-d^{2}$. This inequality, added to the former one, gives $\left|g_{2}-g_{1}\right|^{2}+2 \Re\left[\left(g_{1}-f\right) \times\left(g_{2}-g_{1}\right)\right]$ $\leqq(1 / m)(2 d+1 / m)$. From this, if $\Re\left[\left(g_{1}-f\right) \times\left(g_{2}-g_{1}\right)\right] \geqq 0$, it follows that $\left|g_{2}-g_{1}\right| \leqq((1 / m)(2 d+1 / m))^{1 / 2}$. If $\Re\left[\left(g_{1}-f\right) \times\left(g_{2}-g_{1}\right)\right]<0$ but $\Re\left[\left(g_{2}-f\right) \times\left(g_{1}-g_{2}\right)\right] \geqq 0$, we may reach the same result by interchanging $g_{1}$ and $g_{2}$.

\footnotetext{
I I am using $f \times g$ instead of $(f, g)$.
} 
Let now $\Re\left[\left(g_{1}-f\right) \times\left(g_{2}-g_{1}\right)\right]<0$ and $\Re\left[\left(g_{2}-f\right) \times\left(g_{1}-g_{2}\right)\right]<0$, and put $g_{3}=g_{1}+\alpha\left(g_{2}-g_{1}\right)$, where

$$
0<\alpha=\frac{-\Re\left[\left(g_{1}-f\right) \times\left(g_{2}-g_{1}\right)\right]}{\left|g_{2}-g_{1}\right|^{2}} .
$$

We may also put

$$
0<\beta=\frac{-\Re\left[\left(g_{2}-f\right) \times\left(g_{1}-g_{2}\right)\right]}{\left|g_{1}-g_{2}\right|^{2}},
$$

and so $\alpha+\beta=1, g_{3}=g_{2}+\beta\left(g_{1}-g_{2}\right)$. From the comparison of these two expressions obtained for $g_{3}$, we are enabled to say that every equality or inequality involving $\alpha, \beta, g_{1}, g_{2}, g_{3}$ will still remain true after having interchanged $\alpha$ with $\beta, g_{1}$ with $g_{2}$. Now, we have:

$$
\begin{aligned}
\left|g_{3}-f\right|^{2}= & \left|g_{1}-f\right|^{2}+\alpha^{2}\left|g_{2}-g_{1}\right|^{2}+2 \alpha \Re\left[\left(g_{1}-f\right) \times\left(g_{2}-g_{1}\right)\right] \\
= & \left|g_{1}-f\right|^{2}+\frac{\Re^{2}\left[\left(g_{1}-f\right) \times\left(g_{2}-g_{1}\right)\right]}{\left|g_{2}-g_{1}\right|^{2}} \\
& \quad-2 \frac{\Re^{2}\left[\left(g_{1}-f\right) \times\left(g_{2}-g_{1}\right)\right]}{\left|g_{2}-g_{1}\right|^{2}} \\
= & \left|g_{1}-f\right|^{2}-\frac{\Re^{2}\left[\left(g_{1}-f\right) \times\left(g_{2}-g_{1}\right)\right]}{\left|g_{2}-g_{1}\right|^{2}} \\
& <\left|g_{1}-f\right|^{2} \leqq\left(d+\frac{1}{m}\right)^{2},
\end{aligned}
$$

and therefore $g_{3}$ belongs to $\mathfrak{S}_{m}$. Moreover:

$$
\begin{aligned}
\Re L & \left.\left(g_{3}-f\right) \times\left(g_{1}-g_{3}\right)\right] \\
\quad & =\Re\left[\left(g_{1}-f\right) \times\left(g_{1}-g_{3}\right)\right]+\alpha \Re\left[\left(g_{2}-g_{1}\right) \times\left(g_{1}-g_{3}\right)\right] \\
& =\alpha \Re\left[\left(g_{1}-f\right) \times\left(g_{1}-g_{2}\right)\right]+\alpha^{2} \Re\left[\left(g_{2}-g_{1}\right) \times\left(g_{1}-g_{2}\right)\right] \\
& =\alpha\left\{-\Re\left[\left(g_{1}-f\right) \times\left(g_{2}-g_{1}\right)\right]+\Re\left[\left(g_{1}-f\right) \times\left(g_{2}-g_{1}\right)\right]\right\}=0,
\end{aligned}
$$

so that we may apply to $g_{1}$ and $g_{3}$ the result obtained above. Thus $\left|g_{3}-g_{1}\right| \leqq((1 / m)(2 d+1 / m))^{1 / 2}$, and interchanging $g_{1}$ with $g_{2}$, we have $\left|g_{3}-g_{2}\right| \leqq((1 / m)(2 d+1 / m))^{1 / 2}$. Therefore, under any circumstance, $\left|g_{1}-g_{2}\right| \leqq 2((1 / m)(2 d+1 / m))^{1 / 2}$.

We have now shown that the diameter of $\mathfrak{S}_{m}$ converges to 0 when $m \rightarrow \infty$, and that the requirements of Lemma (2) are fulfilled for the sequence $\left\{\Im_{m}\right\}$. The lemma gives an element $g_{0}$ belonging to every $\mathfrak{S}_{m}$, that is, such that $\left|g_{0}-f\right| \leqq d+1 / m$ for $m=1,2, \cdots$. Hence 
$\left|g_{0}-f\right|=d$. It may easily be shown that $|g-f|>d$ if $g \neq g_{0}$, Q.E.D.

(4) Theorem. A closed linear manifold is separable.

Proof. Let $\mathfrak{M}$ be a closed linear manifold, $\left\{f_{n}\right\}$ a sequence dense in $\mathfrak{S}$. For (3) it is possible to find, for each $f_{n}$, a point $g_{0}^{(n)}$ in $\mathfrak{M}$ such that $\left|f_{n}-g_{0}^{(n)}\right|<\left|f_{n}-g\right|$ for every $g \neq g_{0}^{(n)}$ of $\mathfrak{M}$. We want to show that $\left\{g_{0}^{(n)}\right\}$ is dense in $\mathfrak{M}$. In fact, for every $g$ in $\mathfrak{M}$ and $\epsilon>0$, there exists an integer $n$ such that $\left|g-f_{n}\right|<\epsilon / 2$. Then $\left|g_{0}^{(n)}-f_{n}\right| \leqq\left|g-f_{n}\right|$ $<\epsilon / 2,\left|g_{0}^{(n)}-g\right| \leqq\left|g_{0}^{(n)}-f_{n}\right|+\left|f_{n}-g\right|<\epsilon$, Q.E.D.

When the closed linear manifold $\mathfrak{M}$ is given, and $\left\{g_{0}^{(n)}\right\}$ is built according to the proof of (4), let $\left\{\phi_{n}\right\}$ be the orthonormal set attached to $\left\{g_{0}^{(n)}\right\} .\left\{\phi_{n}\right\}$ determines the closed linear manifold $\mathfrak{M}$, and will be said to be attached to $\mathfrak{M}$.

Now we are able to prove the fundamental theorem:

(5) THEOREM. Let $\mathfrak{M}_{1}, \mathfrak{M}_{2}$ be closed linear manifolds, and $\mathfrak{M}_{1} \subset \mathfrak{M}_{2}$; then the orthogonal complement $\mathfrak{M}_{3}=\mathfrak{M}_{2} \ominus \mathfrak{M}_{1}$ (that is, the set of all the elements of $\mathfrak{M}_{2}$ which are orthogonal to every element of $\mathfrak{M}_{1}$ ) is a nonempty closed linear manifold. Furthermore, if $f_{2}$ is a point of $\mathfrak{M}_{2}$, then $f_{2}=f_{1}+f_{3}$, with $f_{1}$ in $\mathfrak{M}_{1}, f_{3}$ in $\mathfrak{M}_{3}$, uniquely determined.

Proof. Let $\left\{\phi_{n}\right\}$ be the orthonormal set attached to $\mathfrak{M}_{1}$, and

$$
f_{1}=\sum_{1}^{\infty}\left(f_{2} \times \phi_{\alpha}\right) \phi_{\alpha}
$$

according to $S$, Theorem $1.7, f_{3}=f_{2}-f_{1}$ is orthogonal to $\mathfrak{M}_{1}$, hence an element of $\mathfrak{M}_{3}$. The other parts of the proof may be omitted, as they are very simple.

Thus the first aim pointed out in the beginning of this paper is reached. We may add, in case $\mathfrak{M}_{2}=\mathfrak{S}$ in (5), that $f_{1}$ is the projection of $f_{3}=f$ on $\mathfrak{M}_{1}$, and that the element $g_{0}$ of (3) is the projection of $f$ on $\mathfrak{M}$.

We now want to prove the following theorem.

(6) THEOREM. If $H$ is a symmetric transformation whose domain is $\mathfrak{S}$, then $H$ is bounded and therefore continuous.

Proof. (I) The proof consists of a deeper development of the one given in $\mathrm{S}$, p. 59. Let $f_{1}, \cdots, f_{m}$ be arbitrary elements of $\mathfrak{S}_{\mathfrak{C}}, \mathfrak{M}_{1}$ the closed linear manifold determined by $f_{1}, \cdots, f_{m}, H f_{1}, \cdots, H f_{m}$, $H^{2} f_{1}, \cdots, H^{2} f_{m}$, and $\mathfrak{M}_{2}=\mathfrak{S} \ominus \mathfrak{M}_{1}$. If $f$ is an arbitrary element of $\mathfrak{M}_{2}$, we have $f \times f_{i}=f \times H f_{i}=H f \times f_{i}=f \times H^{2} f_{i}=H f \times H f_{i}=0(i=1,2$, $\cdots, m)$. Let $\left\{f_{n}^{(1)}\right\}$ be a sequence dense in $\mathfrak{S}$, and $g_{n}$ the projection 
of $f_{n}^{(1)}$ on $\mathfrak{M}_{2}$, and let $g_{n(m)}$ be the first element of $\left\{g_{n}\right\}$ satisfying the relation $\left|H g_{n(m)}\right| \geqq m\left|g_{n(m)}\right|$, when such an element exists. Then $\phi_{m+1}=g_{n(m)} /\left|g_{n(m)}\right|$ is normal, and $\phi_{m+1}, H \phi_{m+1}$ are orthogonal to $f_{1}, \cdots, f_{m}, H f_{1}, \cdots, H f_{m}$.

(II) If an arbitrary normal element $\phi_{1}$ is chosen, let us put $m=1$, $f_{1}=\phi_{1}$ in (I), and determine, if possible, an element $\phi_{2}$ with the method outlined in (I). Then put $m=2, f_{1}=\phi_{1}, f_{2}=\phi_{2}$, and determine a $\phi_{3}$, and so on, as far as possible. Two cases may occur, which we are going to discuss separately.

Case A: the process may be carried on indefinitely. When this is the case, the elements which have been determined form an orthonormal set $\left\{\phi_{n}\right\}$ with cardinal number $\boldsymbol{\aleph}_{0}$, and such that

$$
H \phi_{i} \times H \phi_{k}=0 \text { if } i \neq k, \quad\left|H \phi_{k}\right| \geqq k .
$$

If we set

$$
h_{n}=\sum_{1}^{n}{ }_{\alpha} \frac{1}{\alpha} \phi_{\alpha}
$$

the sequence $\left\{h_{n}\right\}$ converges to some $h$, according to S, Theorem 1.6, and we have:

$$
\begin{aligned}
|H h|^{2} & =h \times H^{2} h=\lim _{n \rightarrow \infty}\left(h_{n} \times H^{2} h\right), \\
h_{n} \times H^{2} h & =H^{2} h_{n} \times h=\sum_{1}^{n} \sum_{1}^{\infty} \frac{1}{\alpha \beta} H^{2} \phi_{\alpha} \times \phi_{\beta} \\
& =\sum_{1}^{n} \sum_{1}^{\infty} \frac{1}{\alpha \beta} H \phi_{\alpha} \times H \phi_{\beta}=\sum_{1}^{n} \frac{1}{\alpha^{2}}\left|H \phi_{\alpha}\right|^{2} \geqq n,
\end{aligned}
$$

so that $|H h|^{2} \geqq n$ for every $n$. This is absurd.

Case B: the process cannot be carried on indefinitely. If this is the case, there exists an orthonormal set $\phi_{1}, \cdots, \phi_{m}$ which determines a closed linear manifold $\mathfrak{M}_{1}$ such that, if $\mathfrak{M}_{2}=\mathfrak{S} \ominus \mathfrak{M}_{1}$, the projections $g_{n}^{(2)}(n=1,2, \cdots)$ of the elements $f_{n}^{(1)}$ on $\mathfrak{M}_{2}$ satisfy the limitations $\left|H g_{n}^{(2)}\right|<m\left|g_{n}^{(2)}\right|$. If $f$ is an arbitrary element of $\mathfrak{M}_{1}$, we have

$$
\begin{aligned}
f & =x_{1} \phi_{1}+\cdots+x_{m} \phi_{m}, \quad|f|^{2}=\left|x_{1}\right|^{2}+\cdots+\left|x_{m}\right|^{2} \\
|H f|^{2} & =\sum_{1}^{m}{ }_{\alpha, \beta} x_{\alpha} \bar{x}_{\beta} H \phi_{\alpha} \times H \phi_{\beta} \leqq P \sum_{1, \beta}^{m}\left|x_{\alpha}\right|\left|x_{\beta}\right| \\
& \leqq \frac{1}{2} P \sum_{1}^{m}{ }_{\alpha, \beta}\left(\left|x_{\alpha}\right|^{2}+\left|x_{\beta}\right|^{2}\right)=\frac{m+1}{2} P|f|^{2}
\end{aligned}
$$


where $P$ is a suitable positive number. If $g_{n}^{(1)}$ is the projection of $f_{n}^{(1)}$ on $\mathfrak{M}_{1}$, we have

$$
\begin{aligned}
f_{n}^{(1)} & =g_{n}^{(1)}+g_{n}^{(2)}, & & \left|f_{n}^{(1)}\right|^{2}=\left|g_{n}^{(1)}\right|^{2}+\left|g_{n}^{(2)}\right|^{2}, \\
\left|g_{n}^{(1)}\right| & \leqq\left|f_{n}^{(1)}\right|, & & \left|g_{n}^{(2)}\right| \leqq\left|f_{n}^{(1)}\right|,
\end{aligned}
$$

and

$$
\begin{aligned}
\left|H f_{n}^{(1)} \times f_{n}^{(1)}\right| & =\left|\sum_{1}^{2}{ }_{\alpha, \beta} H g_{n}^{(\alpha)} \times g_{n}^{(\beta)}\right| \leqq \sum_{1}^{2}{ }_{\alpha, \beta}\left|H g_{n}^{(\alpha)} \times g_{n}^{(\beta)}\right| \\
& \leqq \sum_{1}^{2}{ }_{\alpha, \beta}\left|H g_{n}^{(\alpha)}\right|\left|g_{n}^{(\beta)}\right| \leqq \sum_{1}^{2} K_{\alpha, \beta}\left|g_{n}^{(\alpha)}\right|\left|g_{n}^{(\beta)}\right| \\
& \leqq \frac{1}{2}\left(K_{1}+K_{2}\right) \sum_{1}^{2}{ }_{\alpha, \beta}\left(\left|g_{n}^{(\alpha)}\right|^{2}+\left|g_{n}^{(\beta)}\right|^{2}\right) \\
& =\frac{3}{2}\left(K_{1}+K_{2}\right)\left|f_{n}^{(1)}\right|^{2},
\end{aligned}
$$

where $K_{1}=((m+1) / 2) P, K_{2}=m$. If we set $3\left(K_{1}+K_{2}\right) / 2=K$, the last inequality may be written $H f_{n}^{(1)} \times\left. f_{n}^{(1)}|\leqq K| f_{n}^{(1)}\right|^{2}$.

If we repeat the process replacing $H$ with $H^{2}$, and introducing a new constant $Q^{2}$, we obtain:

$$
\begin{array}{rlrl}
\left|H^{2} f_{n}^{(1)} \times f_{n}^{(1)}\right| & =\left|H f_{n}^{(1)} \times H f_{n}^{(1)}\right| \leqq Q^{2}\left|f_{n}^{(1)}\right|^{2}, \\
\left|H f_{n}^{(1)}\right| & \leqq Q\left|f_{n}^{(1)}\right| & (Q>0) .
\end{array}
$$

Now let $f$ be an arbitrary element of $\mathfrak{S}$. We have $\left|f_{n}^{(1)} \times H f\right|$ $=\left|H f_{n}^{(1)} \times f\right| \leqq\left|H f_{n}^{(1)}\right||f| \leqq Q\left|f_{n}^{(1)}\right||f|$, and if $g$ is another arbitrary element, and $\epsilon>0$, there exists an integer $n$ such that $\left|g-f_{n}^{(1)}\right|<\epsilon$. It follows that

$$
\begin{aligned}
|g \times H f| & \leqq\left|f_{n}^{(1)} \times H f\right|+\left|\left(g-f_{n}^{(1)}\right) \times H f\right| \\
& \leqq Q\left|f_{n}^{(1)}\right||f|+\left|g-f_{n}^{(1)}\right||H f| \\
& <Q\left|f_{n}^{(1)}\right||f|+\epsilon|H f| \\
& \leqq Q|g||f|+Q\left|f_{n}^{(1)}-g\right||f|+\epsilon|H f| \\
& <Q|g||f|+\epsilon(Q|f|+|H f|),
\end{aligned}
$$

and thus that $|g \times H f| \leqq Q|g||f|$. With this result, and by $\mathrm{S}$, Theorems $2.21,2.22$, the proof is completed, Q.E.D.

Pisa, Italy 